\title{
EXISTENCES AND BOUNDARY BEHAVIOR OF BOUNDARY BLOW-UP SOLUTIONS TO QUASILINEAR ELLIPTIC SYSTEMS WITH SINGULAR WEIGHTS
}

\author{
QIAOYU TIAN AND SHUIBO HUANG *
}

\begin{abstract}
Using the method of explosive sub and supper solution, the existence and boundary behavior of positive boundary blow up solutions for some quasilinear elliptic systems with singular weight function are obtained under more extensive conditions.
\end{abstract}

\section{INTRODUCTION AND MAIN RESULTS}

In this paper, we consider the existence and asymptotic behavior of positive solution to the following elliptic system

$$
\begin{cases}\operatorname{div}\left(|\nabla u|^{m-2} \nabla u\right)=a(x) u^{p} v^{q}, & x \in \Omega, \\ \operatorname{div}\left(|\nabla v|^{n-2} \nabla v\right)=b(x) u^{r} v^{s}, & x \in \Omega,\end{cases}
$$

subject to the boundary conditions

$$
u=v=\infty, \quad x \in \partial \Omega .
$$

where $p>m-1, s>n-1, q, r>0, m, n>1,(p-m+1)(s-n+1)-q r>0, \Omega$ is bounded $C^{2}$ domain of $R^{N}, N \geq 1$, and the last condition (1.2) $u=v=\infty, x \in$ $\partial \Omega$ means that $u \rightarrow \infty, v \rightarrow \infty$ as $d(x):=\operatorname{dist}(x, \partial \Omega) \rightarrow 0$, and the solution is called a large solutions or boundary blow-up solution. By a positive boundary blow-up solutions of (1.1), we mean that $(u, v) \in W_{\mathrm{loc}}^{1, p}(\Omega) \cap C_{\mathrm{loc}}^{1}(\Omega)$ and $(u, v)$ satisfies

$$
\begin{gathered}
-\int_{\Omega}|\nabla u|^{m-2} \nabla u \nabla \varphi d x=\int_{\Omega} a(x) u^{p} v^{q} \varphi d x, \forall \varphi \in C_{0}^{\infty}(\Omega) . \\
-\int_{\Omega}|\nabla v|^{n-2} \nabla v \nabla \varphi d x=\int_{\Omega} b(x) u^{r} v^{s} \varphi d x, \forall \varphi \in C_{0}^{\infty}(\Omega) .
\end{gathered}
$$

Date: Received: 07 April 2009; Revised: 18 April 2009.

* Corresponding author.

2000 Mathematics Subject Classification. 35J60, 35B25, 35B50.

Key words and phrases. Quasilinear elliptic systems; Boundary blow up; Explosive sub- and super-solution. 
and $u, v>0$ in $\Omega, u \rightarrow \infty, v \rightarrow \infty$ as $d(x) \rightarrow 0$,

The study of the elliptic systems is a classical topic that has attracted the attention of many researchers because of its interest in applications, which arises in the theory of quasi-regular and quasi-conformal mappings as well as in the study of non-Newtonian fluids, in non-Newtonian fluids, the pair $(m, n)$ is a characteristic of the medium. Media with $(m, n)>(2,2)$ are called dilatant fluids and those with $(m, n)<(2,2)$ are called pseudoplastics. If $(m, n)=(2,2)$, they are Newtonian fluids.

There is a large amount of literature on elliptic problems related to problem

$$
\Delta u=b(x) f(u), x \in \Omega,\left.x\right|_{\partial \Omega}=\infty .
$$

for $b(x)=1, f(u)=e^{u}$, the problem (1.3) was initiated by Bieberbach [1] for $\Omega \subset R^{2}$. Rademacher[26] extended the results of Bieberbach to $\Omega \subset R^{3}$. Later, Lazer and McKenna [21] generalized the results to the case of bounded domains in $R^{N}$ and nonlinearities $b(x) e^{u}$. For $b \in C_{l o c}^{\alpha}(\Omega), b>0$ in $\Omega$, and provided that $b$ satisfies the following assumption: there exist constants $C_{1}, C_{2}>0, \kappa_{2} \geq \kappa_{1}>-2$ such that

$$
C_{2}(d(x))^{\kappa_{2}} \leq b(x) \leq C_{1}(d(x))^{\kappa_{1}}
$$

and $f(u)$ satisfies: $f \in C^{1}(R)$ is non-decreasing on $R, f(s) \leq C_{1} e^{p_{1} s}$ for all $s \in R$ and $f(s) \geq C_{2} e^{p_{2} s}$ for large $|s|$ with $p_{1} \geq p_{2}>0, C_{1}, C_{2}$ are positive constants, García-Melián[13] showed that problem (1.3) has at least one solution $u \in C^{2}(\Omega)$ such that

$$
\left.-m-\left(2+\gamma_{1}\right) / p_{1} \ln d(x)\right) \leq u(x) \leq M-\left(2+\gamma_{2}\right) / p_{2} \ln d(x), \quad \forall x \in \Omega .
$$

where $m, M$ are positive constants. Very recently, Zhang [33] and Yang[23] extended the above results to the problem (1.3) and gained some new results with nonlinear gradient terms. Problem (1.3) was discussed in a number of works; see, $[2,3,4,5,9,10,11,12,13,19,23,25,34]$,

Now let us return to problem (1.1).

When $m=n=2$, system (1.1) becomes

$$
\begin{cases}\Delta u=a(x) u^{p} v^{q}, & x \in \Omega \\ \Delta v=b(x) u^{r} v^{s}, & x \in \Omega\end{cases}
$$

in the paper [14], when $a(x)=1, b(x)=1$, under Dirichlet boundary conditions of three different types: both components of $(u, v)$ are bounded on $\partial \Omega$ (finite case); one of them is bounded while the other blows up(semilinear case); or both components blow up simultaneously(infinite case), under the assumption that $(a-1)(e-1)>b c$, necessary and suffcient conditions for existence of positive solutions were found, and uniqueness or multiplicity were also obtained, together with the exact boundary behavior of solutions. In addition, they also treated some existence uniqueness and boundary behavior of solutions of systems (1.4) under the assumption

$$
a(x) \sim C_{1} d(x)^{\kappa_{1}}, b(x) \sim C_{2} d(x)^{\kappa_{2}}
$$


when $d(x) \rightarrow 0$ for some positive constants $C_{1}, C_{2}$ and real numbers $\kappa_{1}, \kappa_{2}>-2$. Problem(1.4) was later studied in [15] with general form

$$
C_{1} d(x)^{\kappa_{1}} \leq a(x) \leq C_{2} d(x)^{\kappa_{1}}, C_{3} d(x)^{\kappa_{2}} \leq b(x) \leq C_{4} d(x)^{\kappa_{2}}
$$

for $x \in \Omega$, where $a(x), b(x) \in C^{\theta}(\Omega)$ for some $\theta \in(0,1), \kappa_{1}, \kappa_{2}>-2$, and $C_{i}, i=1,2,3,4$, are positive constants. If the weights $a(x)$ and $b(x)$ satisfy the following two hypotheses:

(I) $a(x) \in C^{\eta}(\Omega), b(x) \in C^{\eta}(\Omega), \eta \in(0,1), a(x)>0, b(x)>0$;

(II) there exist constants $C_{i}>0, i=1,2,3,4$ and $\kappa_{1} \geq \kappa_{2}>-m, \kappa_{3} \geq \kappa_{4}>$ $-n$ such that, for $x \in \Omega$,

$$
C_{1} d(x)^{\kappa_{1}} \leq a(x) \leq C_{2} d(x)^{\kappa_{2}}, C_{3} d(x)^{\kappa_{3}} \leq b(x) \leq C_{4} d(x)^{\kappa_{4}} \quad x \in \Omega,
$$

Let us mention that under the hypothesis (I) and (II), the weight functions $a(x)$ and $b(x)$ may be singular near the boundary $\partial \Omega$. Huang [20] showed that problem (1.4) has unique large solution if and only if $\kappa_{i} \in R, \kappa_{1} \geq \kappa_{2}>-m, \kappa_{3} \geq \kappa_{4}>-n$ and

$$
\frac{q}{s-n+1}<\frac{m+\kappa_{1}}{n+\kappa_{4}}, \frac{m+\kappa_{2}}{n+\kappa_{3}}<\frac{p-m+1}{r} .
$$

the solution verifies

$$
D_{1} d(x)^{-\alpha_{1}} \leq u(x) \leq D_{2} d(x)^{-\alpha_{2}}, D_{3} d(x)^{-\beta_{1}} \leq v(x) \leq D_{4} d(x)^{-\beta_{2}} .
$$

where $D_{i}(i=1,2,3,4)$ are positive constants , and

$$
\begin{gathered}
\alpha_{1}=\frac{\left(m+\kappa_{2}\right)(s-n+1)-\left(n+\kappa_{3}\right) q}{(p-m+1)(s-n-1)-q r}, \alpha_{2}=\frac{\left(m+\kappa_{1}\right)(s-1)-\left(n+\kappa_{4}\right) q}{(p-m+1)(s-n-1)-q r}, \\
\beta_{1}=\frac{\left(m+\kappa_{3}\right)(p-m+1)-\left(n+\kappa_{2}\right) r}{(p-m+1)(s-n-1)-q r}, \beta_{1}=\frac{\left(n+\kappa_{4}\right)(p-m+1)-\left(m+\kappa_{1}\right) r}{(p-m+1)(s-n-1)-q r} .
\end{gathered}
$$

Recently, Yang [31] studied the systems (1.1) and showed that if $a(x)=b(x)=$ $1, p>m-1, s>n-1, q, r>0, m, n>1,(p-m+1)(s-n+1)-q r>0$, then systems (1.1) have boundary blow up solutions, and there exist constants $A, B$ such that

$$
A d(x)^{-\alpha} \leq u(x) \leq B d(x)^{-\alpha}, A d(x)^{-\beta} \leq v(x) \leq B d(x)^{-\beta} .
$$

where,

$$
\alpha=\frac{m(s-q-n+1)}{(p-m+1)(s-n+1)-q r}, \beta=\frac{n(p-r-m+1)}{(p-m+1)(s-n+1)-q r} .
$$

Furthermore, they also obtained the existence and boundary behavior of solutions if $a(x), b(x)$ satisfies $a(x) \sim C_{1} d(x)^{\kappa_{1}}, b(x) \sim C_{2} d(x)^{\kappa_{2}}, \kappa_{1}>-m, \kappa_{2}>-n$.

More results to system with boundary blow up, we refer reader to $[6,7,8,16$, $17,18,22,24,28,29,31,32]$ and references therein.

The main purpose of the present paper is to investigate the influence of the weights $a(x)$ and $b(x)$ on the existence and boundary behavior of solutions of systems (1.1).

The main results of the present paper are the following. 
Theorem 1.1. Assume that $a(x), b(x)$ satisfy (I) and (II), $p>m-1, s>n-1$, $m>1, n>1,(p-m+1)(s-n+1)-q r>0$, Then systems (1.1) has at least a positive solution $(u, v)$ if and only if $\kappa_{i} \in R, \kappa_{1} \geq \kappa_{2}>-m, \kappa_{3} \geq \kappa_{4}>-n$ and

$$
\frac{q}{s-n+1}<\frac{m+\kappa_{1}}{n+\kappa_{4}}, \frac{m+\kappa_{2}}{n+\kappa_{3}}<\frac{p-m+1}{r} .
$$

This solution verifies

$$
D_{1} d(x)^{-\alpha_{1}} \leq u(x) \leq D_{2} d(x)^{-\alpha_{2}}, D_{3} d(x)^{-\beta_{1}} \geq v(x) \geq D_{4} d(x)^{-\beta_{2}} .
$$

where $D_{i}(i=1,2,3,4)$ are positive constants, and

$$
\begin{aligned}
& \alpha_{1}=\frac{\left(m+\kappa_{2}\right)(s-n+1)-\left(n+\kappa_{3}\right) q}{(p-m+1)(s-n-1)-q r}, \alpha_{2}=\frac{\left(m+\kappa_{1}\right)(s-n+1)-\left(n+\kappa_{4}\right) q}{(p-m+1)(s-n-1)-q r}, \\
& \beta_{1}=\frac{\left(m+\kappa_{3}\right)(p-m+1)-\left(n+\kappa_{2}\right) r}{(p-m+1)(s-n-1)-q r}, \beta_{2}=\frac{\left(n+\kappa_{4}\right)(p-m+1)-\left(m+\kappa_{1}\right) r}{(p-m+1)(s-n-1)-q r} .
\end{aligned}
$$

As a straight forward consequence, we obtain

Corollary 1.2. If $d(x) \rightarrow 0, a(x) \sim C_{1} d(x)^{\kappa_{1}}, b(x) \sim C_{2} d(x)^{\kappa_{2}}$ then systems (1.1) have at least a positive solution $(u, v)$ if and only if $\kappa_{i} \in R, \kappa_{1}>-m, \kappa_{2}>$ $-n$ and

$$
\frac{q}{s-n+1}<\frac{m+\kappa_{1}}{n+\kappa_{2}}<\frac{p-m+1}{r} .
$$

This solution verifies

$$
D_{1} d(x)^{-\alpha} \leq u(x) \leq D_{2} d(x)^{-\alpha}, D_{3} d(x)^{-\beta} \leq v(x) \leq D_{4} d(x)^{-\beta} .
$$

where $D_{i}(i=1,2,3,4)$ are positive constants, and

$$
\alpha=\frac{\left(m+\kappa_{1}\right)(s-n+1)-\left(n+\kappa_{2}\right) q}{(p-m+1)(s-n-1)-q r}, \beta=\frac{\left(m+\kappa_{2}\right)(p-m+1)-\left(n+\kappa_{1}\right) r}{(p-m+1)(s-n-1)-q r} .
$$

\section{Proof of TheOrem 1.1}

We now ready to prove Theorem 1.1, whose proof will be split in several lemma. we begin by showing the definitions of blow up supper and subsolutions to systems (1.1).

Definition 2.1. $(\bar{u}, \bar{v}) \in\left(C^{2}(\Omega)\right)^{2}$, is called blow up upper solution of systems (1.1), provided that

$$
\begin{cases}\operatorname{div}\left(|\nabla \bar{u}|^{m-2} \nabla \bar{u}\right) \leq a(x) \bar{u}^{p} \bar{v}^{q}, & x \in \Omega, \\ \operatorname{div}\left(|\nabla \bar{v}|^{n-2} \nabla \bar{v}\right) \geq b(x) \bar{u}^{r} \bar{v}^{s}, & x \in \Omega, \\ \bar{u}=\bar{v}=\infty, & x \in \partial \Omega,\end{cases}
$$

As always, a blow up subsolution $(\underline{u}, \underline{v})$ is defined by reversing the inequalities.

We now recall some already know results which will be used in the proof of Theorem 1.1. Consider

$$
\operatorname{div}\left(|\nabla u|^{m-2} \nabla u\right)=d(x)^{\kappa} u^{p}, x \in \Omega, u=\infty, x \in \partial \Omega,
$$


Here $d(x)$ stands for the distance of a point $x \in \Omega$ to the boundary $\partial \Omega$. This problem has been recently considered in [11], where all issues concerning existence, uniqueness and asymptotic behavior near the boundary of positive solutions were obtained. The following Lemma 2.1 contains the basic feature of problem (2.1), refer the reader to [11] for a proof.

Lemma 2.2. Assume that $p>m-1$ and $\kappa>-m$, then problem (2.1) has a unique positive solution, which will be denoted by $U_{m, p, \kappa}$. Moreover,

$$
D_{1} d(x)^{-\alpha} \leq U_{m, p, \kappa} \leq D_{2} d(x)^{-\alpha},
$$

where $D_{1}, D_{1}>0, \alpha=(m+\kappa) /(p-m+1)$.

Lemma 2.3. Assume that $(\bar{u}, \bar{v}) \in\left(C^{2}(\Omega)\right)^{2},(\underline{u}, \underline{v}) \in\left(C^{2}(\Omega)\right)^{2}$ are upper solution and subsolution of systems

$$
\begin{cases}\operatorname{div}\left(|\nabla u|^{m-2} \nabla u\right)=a(x) u^{p} v^{q}, & x \in \Omega \\ \operatorname{div}\left(|\nabla v|^{n-2} \nabla v\right)=b(x) u^{r} v^{s}, & x \in \Omega \\ u=f(x), v=g(x), & x \in \partial \Omega\end{cases}
$$

and $\underline{u} \leq f(x) \leq \bar{u}, \underline{v} \geq g(x) \geq \bar{v}, x \in \partial \Omega, \underline{u} \leq \bar{u}, \underline{v} \geq \bar{v}, x \in \Omega$, here, f, $g \in$ $C^{\eta}(\Omega)(\eta \in(0,1))$. Then systems (2.3) has at least a solution $(u, v)$, and $\underline{u} \leq u \leq$ $\bar{u}, \underline{v} \geq v \geq \bar{v}, x \in \Omega$, in particular, $u=f(x), v=g(x), x \in \partial \Omega$.

Proof. Let $u_{1}$ (the existence and uniqueness of $u_{1}$ see Remark 3 of [11]) is the unique positive solution of

$$
\operatorname{div}\left(|\nabla u|^{m-2} \nabla u\right)=a(x) \underline{v}^{q} u^{p}, \quad x \in \Omega, u=f(x), \quad x \in \partial \Omega,
$$

Clearly, $\bar{u}$ and $\underline{u}$ are the upper solution and subsolution of (2.4), thanks to uniqueness of $u_{1}$, we have $\bar{u} \leq u_{1} \leq \underline{u}$. now assume that $v_{1}$ is the unique positive solution of

$$
\operatorname{div}\left(|\nabla u|^{n-2} \nabla u\right)=b(x) u_{1}^{r} v^{s}, x \in \Omega, v=g(x), x \in \partial \Omega,
$$

It following similarly that $\bar{v} \geq v_{1} \geq \underline{v}$. We can continue in this way by defining $u_{n}$ to be the unique solution to (2.4) whit $v$ replaced by $v_{n-1}$ and $v_{n}$ the unique solution to $(2.5)$ with $u_{1}$ replaced by $u_{n}$. We obtain unique positive solution sequences $\left\{u_{n}\right\},\left\{v_{n}\right\}$, such that $\underline{u} \leq u_{n} \leq \bar{u}, \underline{v} \geq v_{n} \geq \bar{v}$. Moreover, $u_{n}$ is increasing and $v_{n}$ is decreasing. From standard regularity and compactly embedding theory, it following that there exist subsequence $\left\{u_{n_{k}}\right\},\left\{v_{n_{k}}\right\}$, such that $u_{n_{k}} \rightarrow u(x), v_{n_{k}} \rightarrow v(x), x \in C^{\eta}(\bar{\Omega}) \bigcap C_{l o c}^{1}(\Omega)$, where $\{u(x), v(x)\}$ is the solution of (2.3), moreover, $u(x)=f(x), v(x)=g(x)$ and $\underline{u} \leq u \leq \bar{u}, \bar{v} \geq v \geq \underline{v}$.

Lemma 2.4. Set $(\bar{u}, \bar{v})$ and $(\underline{u}, \underline{v})$ are blow up upper solution and subsolution of (1.1) with $\underline{u}=\bar{u}=\underline{v}=\bar{v}=\infty, x \in \partial \Omega$ and $\underline{u} \leq \bar{u}, \underline{v} \geq \bar{v}, x \in \Omega$. Then systems (1.1) $+(1.2)$ have at least positive solution $(u, v)$ such that $\underline{u} \leq u \leq \bar{u}$, $\underline{v} \geq v \geq \bar{v}, x \in \Omega$, in particular, $u=v=\infty, x \in \partial \Omega$.

Proof. Set $\Omega_{\delta}=\{x \in \Omega: d(x)>\delta\}$, where $\delta>0, d(x):=\operatorname{dist}(x, \partial \Omega)$, and consider the problem

$$
\begin{cases}\operatorname{div}\left(|\nabla u|^{m-2} \nabla u\right)=a(x) u^{p} v^{q}, & x \in \Omega_{\delta}, \\ \operatorname{div}\left(|\nabla v|^{n-2} \nabla v\right)=b(x) u^{r} v^{s}, & x \in \Omega_{\delta}, \\ u=f_{\delta}, v=g_{\delta}, & x \in \partial \Omega_{\delta},\end{cases}
$$


where $f_{\delta}, g_{\delta}$ are are smooth functions defined on $\partial \Omega_{\delta}$ with $\underline{u} \leq f_{\delta} \leq \bar{u}, \underline{v} \geq$ $g_{\delta} \geq \bar{v}, x \in \Omega$, in view of Lemma 2.2 and standard regularity theory, it following that systems (2.6) have a bounded solution $\left(u_{\delta}, v_{\delta}\right)$ in $C_{\mathrm{loc}}^{1, \eta}(\Omega)$, so that we obtain subsequence $\left(u_{\delta_{k}}, v_{\delta_{k}}\right) \rightarrow(u, v), x \in C_{l o c}^{1}(\Omega)$, where $(u, v)$ is a positive solution of (1.1) with $\underline{u} \leq u \leq \bar{u}, \underline{v} \geq v \geq \bar{v}, x \in \Omega$ and $u=v=\infty, x \in \partial \Omega$.

Lemma 2.5. Assume $\kappa_{i} \in R, \kappa_{1} \geq \kappa_{2}>-m, \kappa_{3} \geq \kappa_{4}>-n$ and (1.6) holds, Then system (1.1)+(1.2) admits at least one positive solution.

Proof. We use the method of blow up sub and super solution. Let $(\underline{u}, \underline{v})=$ $\left(\varepsilon U_{m, p, \tau_{1}}, \varepsilon^{-\delta} U_{n, s, \delta_{1}}\right)$, where the functions $U_{m, p, \tau_{1}}, U_{n, s, \delta_{1}}$ are as introduced above, $\varepsilon$ is small enough, $\tau_{1}, \delta_{1}, \delta$ are to be chosen such that $(\underline{u}, \underline{v})$ is the blow up sub solution of system (1.1).

Combining with (2.1), (2.2) and the definition of blow up sub and super solution, if we select

$$
\begin{gathered}
\tau_{1}=(p-m+1) \alpha_{1}-m>-m, \delta_{1}=(s-n+1) \beta_{1}-n>-n, \\
r /(s-n+1)<\delta<(p-m+1) / q .
\end{gathered}
$$

$\alpha_{1}, \beta_{1}$ are gave by (1.8) and (1.9). A simple calculation show that

$$
\begin{aligned}
& \operatorname{div}\left(\left|\nabla\left(\varepsilon U_{m, p, \tau_{1}}\right)\right|^{m-2} \nabla\left(\varepsilon U_{m, p, \tau_{1}}\right)\right)=\varepsilon^{m-1} \operatorname{div}\left(\left|\nabla U_{m, p, \tau_{1}}\right|^{m-2} \nabla U_{m, p, \tau_{1}}\right) \\
= & \varepsilon^{m-1} d(x)^{\tau_{1}} U_{m, p, \tau_{1}}^{p} \geq C_{2} d(x)^{\kappa_{2}} \varepsilon^{p-q \delta} U_{m, p, \tau_{1}}^{p} U_{n, s, \delta_{1}}^{q}, \\
& \operatorname{div}\left(\left|\nabla\left(\varepsilon^{-\delta} U_{n, s, \delta_{1}}\right)\right|^{n-2} \nabla\left(\varepsilon^{-\delta} U_{n, s, \delta_{1}}\right)\right)=\varepsilon^{-\delta(n-1)} \operatorname{div}\left(\left|\nabla U_{n, s, \delta_{1}}\right|^{n-2} \nabla U_{n, s, \delta_{1}}\right) \\
= & \varepsilon^{-\delta(n-1)} d(x)^{\delta_{1}} U_{n, s, \delta_{1}}^{s} \leq C_{3} d(x)^{\kappa_{3}} \varepsilon^{r-s \delta} U_{m, p, \tau_{1}}^{r} U_{n, s, \delta_{1}}^{s},
\end{aligned}
$$

which leads to $\left(\varepsilon U_{m, p, \tau_{1}}, \varepsilon^{-\delta} U_{n, s, \delta_{1}}\right)$ is the sub solution of system (1.1). similarity, we can select $\tau_{2}=(p-m+1) \alpha_{2}-m>-m, \delta_{2}=(s-n+1) \beta_{2}-n>-n, \alpha_{2}, \beta_{2}$ are gave by (1.8) and (1.9), if $M$ is large enough, then $(\bar{u}, \bar{v})=\left(M U_{m, p, \tau_{2}}, M^{-\delta} U_{n, s, \delta_{2}}\right)$ is the super solution of system (1.1).

By $\kappa_{1} \geq \kappa_{2}>-m, \kappa_{3} \geq \kappa_{4}>-n$ and the definition of $\alpha_{i}, \beta_{i}$, obtain $\alpha_{1} \leq \alpha_{2}$, $\beta_{1} \geq \beta_{2}$, then we get $\underline{u} \leq \bar{u}$ and $\underline{v} \geq \bar{v}, x \in \Omega$, according to Lemma 2.3 , there exist a positive solution $(u, v)$ of system (1.1)+(1.2) with $\underline{u} \leq u \leq \bar{u}$ and $\underline{v} \geq v \geq$ $\bar{v}, x \in \Omega$, in particular, $u=v=\infty, x \in \partial \Omega$.

Lemma 2.6. Let $(u, v)$ be a positive solution to system $(1.1)+(1.2)$. Then there exist constants $D_{i}(i=1,2,3,4)$ such that (1.7) holds,

Proof. By the definition of $U_{m, p, \tau_{i}}, U_{n, s, \delta_{i}}$ and (2.2), we infer that there exist positive constants $E_{i}, F_{i}, E_{i}^{\prime}, F_{i}^{\prime}(i=1,2)$ such that

$$
E_{i} d(x)^{-\alpha_{i}} \leq U_{m, p, \tau_{i}} \leq E_{i}^{\prime} d(x)^{-\alpha_{i}}, F_{i} d(x)^{-\beta_{i}} \leq U_{n, s, \delta_{i}} \leq F_{i}^{\prime} d(x)^{-\beta_{i}} .
$$

Since $\varepsilon U_{m, p, \tau_{1}}=\underline{u} \leq u \leq \bar{u}=M U_{m, p, \tau_{2}}, \varepsilon^{-\delta} U_{n, s, \delta_{1}}=\underline{v} \geq v \geq \bar{v}=M^{-\delta} U_{n, s, \delta_{2}}, x \in$ $\Omega$ and $\alpha_{1} \leq \alpha_{2}, \beta_{1} \geq \beta_{2}$, this implies that (1.7) holds.

We finally show that the conditions $\kappa_{i} \in R, \kappa_{1} \geq \kappa_{2}>-m, \kappa_{3} \geq \kappa_{4}>-n$ and (1.6) are necessary for problem $(1.1)+(1.2)$ to have a positive solution.

Lemma 2.7. Assume problem (1.1)+(1.2) has a positive solution $(u, v)$. Then $\kappa_{i} \in R, \kappa_{1} \geq \kappa_{2}>-m, \kappa_{3} \geq \kappa_{4}>-n$ and (1.6) holds. 
Proof. If $\left(m+\kappa_{1}\right) /\left(n+\kappa_{4}\right) \leq q /(s-n+1)$, by the definition of $\alpha_{2}$, we have $\alpha_{2} \leq 0$, then (1.7)implies $u$ is bounded. Similarity, if $\left(m+\kappa_{2}\right) /\left(n+\kappa_{3}\right) \geq(p-m+1) / r$, we also obtain $v$ is bounded by (1.7), which are contradiction to $u=v=\infty, x \in \partial \Omega$. This finishes the proof.

\section{REFERENCES}

[1] L. Bieberbach, $\Delta u=e^{u}$ und die automorphen Funktionen, Math. Ann. 77 (1916), 173-212.

[2] C. Bandle, M. Marcus, "Large" solutions of semilinear elliptic equations: Existence, uniqueness and asymptot behaviour, J. Anal. Math. 58 (1992), 9-24.

[3] T . Boni, D. Nabongo, R. Sery, Blow-up time of some nonlinear wave equations, J. Nonlinear Sci. Appl. 2 (2008), 91-101.

[4] M. Chuaqui, C. Corázar, M. Elgueta, J. García-Melián, Uniqueness and boundary behaviour of large solution elliptic problems with singular weights, Commun.Pure. Appl. Anal.3 (2004), 653-662.

[5] F. Cîrstea, Y. Du, General uniqueness results and variation speed for blow-up solutions of elliptic equations, Proc. London Math. Soc, 91 (2005), 459-482.

[6] N. Dancer, Y. Du, Effects of certain degeneracies in the predator-prey model. SIAM J. Math. Anal. (2002), 292-314.

[7] Y. Du, Effects of a degeneracy in the competition model. Part I: Classical and generalized steady-state solutions. J. Differential Equations. 181 (2002), 92-132.

[8] Y. Du, Effects of a degeneracy in the competition model. Part II: Perturbation and dynamical behaviour. J. Differential Equations. 181 (2002), 133-164.

[9] J. L. Gómez, Optimal uniqueness theorems and exact blow-up rates of large solutions, J. Differential Equations. 224 (2006)' 385-439.

[10] J. L. Gómez, The boundary blow-up rate of large solutions, J. Differential Equations. 195(2003) 25-45.

[11] J. García-Melián, Large solutions for equations involvingt p-Laplacian and singular weights, Z. angew. Math. Phys. (2009).

[12] J. García-Melián, Uniqueness for boundary blow-up poblems with continuous weights, Proc. Amer. Math. Soc. 135 (2007)' 2785-2793.

[13] J. García-Melián, A remark on the existence of positive large solutions via sub and supersolutions, Electron J. Differential Equations. 110 (2003), 1-4.

[14] J. García-Melián, J. D. Rossi, Boundary blow-up solutions to elliptic systems of competitive type, J. Differential Equations. 206 (2004), 156-181.

[15] J. García-Melián, A remark on uniqueness of large solutions for elliptic systems of competitive type, J. Math. Anal. Appl. 331 (2007), 608-616.

[16] J. García-Melián, R. Letelier-Albornoz, J. Sabina de Lis, The solvability of an elliptic system under a singular boundary condition, Proc. Roy. Soc. Edinburgh, 136 (2006), 509546.

[17] J. García-Melián, A. Suárez, Existence and uniqueness of positive large solutions to some cooperative elliptic systems, Adv. Nonlinear Studies, 3 (2003), 193-206.

[18] J. García-Melián, Large solutions for an elliptic system of quasilinear equations, J. Differential Equations. 245 (2008), 3735-3752.

[19] S. Huang, Q. Tian, C. Mu, Asymptotic behavior of large solution to elliptic equation of Bieberbach-Rademacher type with convection terms, Appl. Math. Comput. 210 (2009), 284-293.

[20] S. Huang, Q. Tian, C. Mu, The prospers of boundary blow-up solutions to elliptic systems of competitive type with singular weights, Chinese J. Engrg Math. (in Chinese) (2009) in press.

[21] A. C. Lazer, P. J. McKenna, On a problem of Bieberbach and Rademacher, Nonlinear Anal. 21 (1993), 327-335. 
[22] H. Li, M. Wang, Existence and uniqueness of positive solutions to the boundary blow-up problem for an elliptic system. J. Differential Equations. 234 (2007), 246-266.

[23] C. Liu, D. Yang, Existence of large solutions for a quasilinear elliptic problem via explosive sub-supersolutions, Appl. Math. Comput, 199 (2008), 414-424.

[24] C. Mu, S. Huang, Q. Tian, L. Liu, Large solutions for an elliptic system of competitive type: Existence, uniqueness and asymptotic behavior, Nonlinear Anal. (2009).

[25] McKenna P L, Reichel, Walter W. Symmetry and multiplicity for nonlinear elliptic differential equations with boundwry blow-up. Nonlinear Anal. (1997), 1213-1225.

[26] H. Rademacher, Einige besondere probleme partieller Differentialgleichungen, in Die Differential und Integralgleichungen der Mechanik und Physik, I,Rosenberg, New York, second ed. (1943) 838-845.

[27] S. I. Resnick, Extreme Values, Regular Variation, and Point Processes, Springer Verlag, Berlin/New York, 1987.

[28] L. Wei, M.Wang, Existence and estimate of large solutions for an elliptic system. Nonlinear Anal.70 (2009), 1096-1104.

[29] M.Wang, L. Wei, Existence and boundary blow-up rates of solutions for boundary blow-up elliptic systems, Nonlinear Anal. (2009).

[30] Z. Wu , D. Yang, Existence of boundary blow-up solutions for a class of quasilinear elliptic systems with critical case. Appl Math Comput. 198 (2008) 574-581.

[31] D.Yang, Z. Wu, Existence of boundary blow-up solutions for a class of quasilinear elliptic systems with subcritical case. Commun.Pure. Appl. Anal. 6 (2007), 531-540.

[32] D. Yang, Existence of entire explosive positive radial solutions for a class of quasilinear elliptic systems. J. Math. Anal. Appl. 288 (2003), 768-783.

[33] Z. Zhang, Existence of large solutions for a semilinear elliptic problem via explosive subsupersolutions, Electron J. Differential Equations. 2 (2006), 1-8.

[34] F. Zhang, Multiple positive solutions for nonlinear singular third-order boundary value problem in abstract space, J. Nonlinear Sci. Appl. 1 (2008), 36-44.

Department of Mathematics Hezuo Minorities Teacher College, Hezuo Gansu , 747000. P. R. CHINA

E-mail address: huangshuibo2008@163.com 